\title{
A distinct phenotype characterizes tumors from a putative genetic trait involving chondrosarcoma and breast cancer occurring in the same patient
}

\author{
Anne-Marie Cleton-Jansen ${ }^{1}$, Michel C Timmerman ${ }^{1}$, Marc J van de Vijver ${ }^{1,5}$, Christi J van \\ Asperen $^{2}$, Herman M Kroon ${ }^{3}$, Paul HC Eilers ${ }^{4}$ and Pancras CW Hogendoorn ${ }^{1}$
}

${ }^{1}$ Department of Pathology; ${ }^{2}$ Department of Clinical Genetics; ${ }^{3}$ Department of Radiology; ${ }^{4}$ Department of Medical Statistics, Leiden University Medical Center, Leiden, The Netherlands and ${ }^{5}$ Department of Pathology, The Netherlands Cancer Institute, Antoni van Leeuwenhoek Hospital, Amsterdam, The Netherlands

\begin{abstract}
Recently, we documented an increased risk for the occurrence of breast- and cartilaginous tumors in the same patient, statistically pointing towards a potential genetic trait. This trait is most probably not associated with mutations in the two major hereditary breast cancer genes since no cases of enchondroma or chondrosarcoma were found in Dutch BRCA1 and BRCA2 families. We were able to collect and review the tumor tissue samples from 34 patients with both breast- and cartilaginous tumors and compared histopathological and immunohistochemical features of these tumors with controls. Breast cancer controls were available from literature data generated to compare familial breast cancers with nonselected cases. Clinical markers for chondrosarcoma controls were collected from the Netherlands Committee of Bone Tumors. Immunohistochemical data on chondro-tumor controls were available from our own files. Breast tumors of patients with cartilaginous sarcomas showed a significantly higher mitotic count $(P=0.001)$, contained less lymphocyte infiltrate $(P=0.025)$ and less nuclear pleomorphism. Remarkably, all cartilaginous tumors are of one common histological category originating centrally $(P=0.014)$. Estrogen receptor and p53 expression were significantly higher $(P<0.001)$ in breast cancer associated with chondro-tumors. p21 staining was more often negative in chondro-tumors associated with breast cancer. In seven cases of breast cancer, we found a slight decrease in CHEK2 expression. However, we could not identify the CHEK2 1100delC mutation in these cases nor in cases with normal CHEK2 expression. Hierarchical cluster analysis of all parameters within chondro-tumorassociated breast cancer specimens revealed two different subgroups, the largest one associated with estrogen receptor-positive breast cancer, which may distinguish sporadic cases from those belonging to the potential genetic trait. These distinct phenotypic findings support the existence of a new hitherto unrecognized syndrome, characterized by an increased risk to develop both breast cancer and centrally originating cartilaginous tumors.
\end{abstract}

Laboratory Investigation (2004) 84, 191-202, advance online publication, 8 December 2003; doi:10.1038/labinvest.3700032

Keywords: chondro-tumor; enchondroma; estrogen receptor; hereditary cancer; immunohistochemistry; CHEK2

Recently, we have shown a strong association between the occurrence of cartilaginous tumors and breast cancer in the same patient, using a nation-wide case-control study. ${ }^{1}$ Compared with a group of patients developing lung and breast cancer,

Correspondence: A-M Cleton-Jansen, Department of Pathology, Leiden University Medical Center, PO box 9600, L1-Q, 2300 RC Leiden, The Netherlands.

E-mail: A.M.Cleton-Jansen@lumc.nl

Received 28 August 2003; revised 7 October 2003; accepted 13

October 2003; published online 8 December 2003 this study revealed an odds ratio of 7.62 for a potential association of breast and cartilaginous tumors. This finding is pointing strongly towards a genetic trait, which is furthermore corroborated by the age of onset in patients with breast cancer as their first tumor, that is, about 10 years earlier than breast cancer in the general population.

In general, $5 \%$ of breast cancers are hereditary and a subset of these has been shown to be attributable to germline mutations in BRCA1 and BRCA2. Tumors in BRCA1 and BRCA2 families have characteristic phenotypes. Especially BRCA1 germline mutated 
tumors show striking histological features, for example, the noninfiltrative pushing borders phenomenon, and a characteristic immunohistochemical phenotype. ${ }^{2,3}$ Furthermore, cDNA microarray analysis identified specific gene expression profiles for BRCA1 and BRCA2-associated tumors. ${ }^{4,5}$

Chondrosarcomas are rare malignant cartilageforming tumors occurring principally in adults in the third to sixth decade. These tumors arise centrally in bone (primary or central chondrosarcoma, $75 \%$ of all cases), or within the cartilaginous cap of a formerly benign osteochondroma (secondary or peripheral chondrosarcoma, $15 \%$ of all cases). ${ }^{6-9}$ Recent data have shown that both subtypes are likely to originate due to different genetic mechanisms. ${ }^{10-12}$ Secondary chondrosarcomas often have near-haploidy in low-grade and polyploidization in high-grade peripheral tumors. In contrast, central chondrosarcomas show few genetic aberrations. ${ }^{10}$

Malignant transformation is low in solitary osteochondromas $(<1 \%)$ but is estimated to occur in 1$5 \%$ of cases of multiple osteochondromas (MO), a familial disorder with an autosomal dominant mode of inheritance. ${ }^{13,14}$ Two genes involved in heparansulfate proteoglycan synthesis, EXT1 and EXT2 have been shown to be mutated in MO patients. ${ }^{15}$ The majority of primary or central chondrosarcomas are considered to originate de novo, while a small number arises secondarily from an enchondroma. Enchondromas are benign cartilage-forming tumors arising solitary and multiple as in Ollier's disease and Maffucci's syndrome. So far, no hereditary forms of primary chondrosarcoma have been described.

To characterize this possible breast-cartilaginous cancer trait, we did a survey into the phenotypic characteristics of both chondro- and breast tumors of patients with both tumor types and compared them with their sporadic counterparts. First, we examined the histological characteristics of these tumors to identify a putative specific histological subtype or any other histological features predominating within these breast- and cartilaginous tumors. In addition, we performed immunohistochemical staining on these tumor specimens. The identification of a possible specific protein expression profile and pronounced histological features would further corroborate the existence of a genetic trait, could provide a tool to distinguish sporadic cases from those associated with this genetic trait and would also provide us with more insight into the mechanisms contributing to this putative cancer syndrome.

\section{Material and methods}

\section{Patient Data}

Through the Pathological Anatomy National Automated Archive (PALGA), we obtained data from all 61 patients in the Netherlands who developed both breast cancer and enchondroma/chondrosarcoma between 1973 and 1996. PALGA approached pathology departments in the Netherlands to supply us with formalin-fixed, paraffin-embedded tumor specimens and clinical data of these patients, without revealing the names of the patients, compliant with Dutch regulations on protection of privacy. As a result of the anonymized procedure to collect the patients we had no data on ethnic origin of this group. We did obtain the age of onset of both breast cancer and chondro-tumor.

\section{Control groups for Histology and Immunohistochemistry}

To compare breast cancer specimens associated with cartilaginous tumors, control groups were obtained from the literature both for histology (referred to as control group 1, $n=1463)^{3}$ and for immunohistochemistry (control group 2, $n=204$ ). ${ }^{16}$ Exact data on age of onset of these controls was not available, but these controls were collected for a study on hereditary breast cancer and involve patients with a relatively early age of onset, like in our patient group. One of the pathologists $(\mathrm{MvdV})$, who was involved in these studies was also responsible for the examination of the breast tumors described in the study described here. No appropriate controls with a similar scoring system are available for p16 and p21 immunohistochemical staining.

The histological subtypes within the cartilaginous tumors were compared with 20542 cases of bone cancer obtained from the literature (control group 3). ${ }^{17}$ Grading and tumor localization were compared with histological data from 200 women from the files of the Netherlands Committee on Bone Tumors, all diagnosed with primary cartilaginous sarcomas or enchondromas in the same time frame (control group 4). P21 and P16 immunohistochemical data for nonselected sporadic cartilaginous tumors were generated on 66 primary chondrosarcomas and 43 enchondromas obtained from the Leiden University Medical Center (control group 5) (manuscripts in preparation). For p53 immunohistochemical staining controls were only available for chondrosarcomas (control group 6), not for enchondromas. ${ }^{18}$

\section{Histology}

The breast cancer tissue samples were classified and histologically characterized according to the WHO criteria. ${ }^{19}$ An expert breast pathologist (MvdV) assessed type of invasive cancer, histological grade, presence of a component of carcinoma in situ and the presence of lymphocyte infiltrate. Age of the patient at time of breast cancer diagnosis was available from PALGA.

Histological grading and classification of the cartilaginous tumors was performed according to Evans et $a l^{8,9,20}$ by an expert pathologist on bone tumors (PCWH). The cartilaginous tumors were 
analyzed with emphasis on the central vs peripheral localization in the skeleton. Localization of the cartilaginous tumor and age of the patient at the time of diagnosis of these tumors were also verified.

\section{Immunohistochemistry}

Monoclonal antibodies used in this study and manufacturers are listed in Table 1. Immunohistochemistry was performed as described ${ }^{21}$ with a few adjustments. After dewaxing, endogenous peroxidase was blocked with $0.3 \%$ hydrogen peroxide in $100 \%$ methanol for $20 \mathrm{~min}$. Antigen retrieval was performed for $10 \mathrm{~min}$ in a boiling solution of $0.01 \mathrm{M}$ Citrate buffer $\mathrm{pH}=6$ for all antibodies except for Her2, followed by an overnight incubation with the primary antibodies. Binding of the antibodies was detected with biotinylated rabbit-anti-mouse Ig or biotinylated swine-anti-rabbit Ig (for the progesterone receptor) followed by a biotinylated peroxidase streptavidin complex (DAKO, Glostrup, Denmark). For visualization of peroxidase bound antibody diaminobenzidine (DAB) was used as chromogen. For all assays, selected tissue samples were used as positive control. Corresponding species and isotypespecific IgGs were used as negative controls. All incubations were performed at room temperature and all washings in between were done with PBS. Immunohistochemical results were scored using a semiquantitative method as described previously. ${ }^{22}$ Table 2 lists the four different scoring systems used in this study, which were applied to make our data comparable with those from the control series. Therefore, sometimes different scoring systems were used for the same antibody in breast and chondrotumor samples, for example for p53 (Table 1).

\section{CHEK 2 1100delC Mutation Screening}

The 1100delC variant in CHEK2 was analyzed on genomic DNA extracted from paraffin-embedded formalin-fixed breast tumor tissue, using a Wizard genomic DNA purification kit (Promega, The
Netherlands). The fragment containing the possible variant was PCR amplified in two rounds to avoid amplification of pseudogenes according to a protocol described in Oldenburg et al (in press) with some modifications. The first round of PCR used primer WI-3F, labeled with FAM (5'-TGT CTT CTT GGA CTG GCA GA-3') and CHK2 10R (5'-ATC ACC TCC TAC CAG TCT GTG-3') with Amplitaq Gold (Roche) at 33 cycles and $65^{\circ} \mathrm{C}$ annealing followed by a second round on $1 \mu \mathrm{l}$ first-round PCR product using the same forward primer, WI-3F and WI-3R (5'-GTT TGT TCT CCC AAA ATC TTG GAG TGC-3') at 33 cycles and $59^{\circ} \mathrm{C}$ annealing. WI-3R contains an additional nontemplated $5^{\prime}$ hexamer to avoid stutter bands. A volume of $1 \mu \mathrm{l}$ PCR reaction was mixed with formamide and size marker and run on an ABI 310 automated sequencer (Applied Biosystems). A breast cancer DNA with a known 1100delC mutation was used as a positive control. The heterozygous

Table 2 Immunohistochemical scoring systems

\begin{tabular}{|c|c|c|}
\hline Scoring system & Staining intensity & Percentage of cells stained \\
\hline A & $\begin{array}{l}0=\text { negative } \\
1=\text { low } \\
2=\text { medium } \\
3=\text { high }\end{array}$ & $\begin{array}{l}0=0 \\
1=1-10 \\
2=11-25 \\
3=26-50 \\
4=51-75 \\
5=76-100\end{array}$ \\
\hline $\mathrm{B}$ & $\begin{array}{l}0=\text { negative } \\
1=\text { low } \\
2=\text { medium } \\
3=\text { high }\end{array}$ & $\begin{array}{l}0=0 \\
1=1-25 \\
2=26-50 \\
3=51-75 \\
4=76-100\end{array}$ \\
\hline $\mathrm{C}$ & $\begin{array}{l}0=\text { negative } \\
1=\text { positive }\end{array}$ & \\
\hline $\mathrm{D}$ & & $\begin{array}{l}0=<25 \\
1=\geq 25\end{array}$ \\
\hline $\mathrm{E}$ & $\begin{array}{l}0=\text { negative } \\
1=\text { low } \\
2=\text { high }\end{array}$ & \\
\hline
\end{tabular}

Table 1 Monoclonal antibodies and scoring systems used in immunohistochemistry

\begin{tabular}{|c|c|c|c|c|c|}
\hline Antigen & Clone & Manufacturer & Scoring $B C^{\mathrm{a}}$ & Scoring $C T^{\mathrm{b}}$ & Threshold for positivity \\
\hline P16 & JC8 & Neomarkers, Union City, USA. & $\mathrm{B}$ & $\mathrm{B}$ & $>0$ \\
\hline P16 & $16 \mathrm{P} 04$ & Neomarkers, Union City, USA. & $\mathrm{B}$ & & $>0$ \\
\hline P21 & AB-1 & Calbiochem, Cambridge, USA. & B & $\mathrm{C}$ & $>0$ \\
\hline P53 & DO7 & Neomarkers, Union City, USA. & B & $\mathrm{D}$ & $>0$ \\
\hline Neu-c-erbB-2 & 3B5 & c & $\mathrm{C}$ & & $>0$ \\
\hline Ecadherin & 36 & Transduction Lab., Franklin Lakes, USA. & B & & $>2$ \\
\hline Progesterone receptor & & DAKO a/s, Glostrup, Denmark. & A & & $>2$ \\
\hline Estrogen receptor & 1D5 & DAKO a/s, Glostrup, Denmark. & A & & $>2$ \\
\hline CHEK2 & DCS270.1 & Novocastra & $\mathrm{E}$ & & $>0$ \\
\hline
\end{tabular}

${ }^{\mathrm{a}}$ See Table 2 for scoring systems, $\mathrm{BC}=$ breast cancer.

${ }^{\mathrm{b}} \mathrm{CT}=$ cartilaginous tumor.

“As described bij MJ van der Vijver et al. N Engl J Med 1988;319(19):1239-1245. 
1100delC mutation is detected as an additional peak one basepair smaller than the wildtype peak.

\section{Statistical Analysis}

Statistical analysis was performed using SPSS software. Comparison of the breast- and cartilaginous tumor specimens associated with this syndrome and controls, of both histology and immunohistochemistry, were calculated using the $\chi^{2}$ test. $P$-values of 0.05 or less were interpreted as significant. Because the immunohistochemical stainings for control population of breast cancer patients were not performed in the same laboratory as for our patient series comparisons were limited to two categories with thresholds between positive and negative staining indicated in Table 1 . In addition, a Bonferroni correction was done on the $P$-values to adjust for the number of parameters tested. For this analysis, we used the program on the simple interactive statistical analysis (SISA) website, which calculates the adjusted $P$-value using the number of tests and the correlation between the parameters tested for.

To examine if these syndrome-associated tumors display a characteristic phenotypic profile, we did a hierarchical cluster analysis with all parameters of both histology and immunohistochemistry using the software programs Cluster and TreeView. ${ }^{23}$ For this analysis, we used the different classes of staining intensity and percentage of stained tumor cells. This was done for the combination of both tumors and for each tumor separately. Using the cluster program the data for each histological parameter or immunohistochemical scoring were normalized, mean centered and average linking clustering was applied. For similarity metrics uncentered correlation was used. For all antibodies, except Her2 in breast cancers and p21 in cartilaginous tumors, staining intensity and percentage-positive cells were analyzed separately.

\section{Results}

\section{No Association between Breast Cancer and Osteochondroma}

Our initial search for the association between breastand cartilaginous tumors was aimed at the identification of those cartilaginous lesions that are detected by radiographic screening for metastases, thereby excluding osteochondromas, which are usually not detected by radiological screening procedures. In this study, we have assessed whether the cartilaginous tumors that belong to this putative syndrome are of a particular histological subset, and therefore we had to determine whether there is also an increased risk for osteochondromas. Like in our previous study, which identified an increased relative risk to develop a chondro-tumor in patients with breast cancer and vice versa, ${ }^{1}$ we compared the occurrence of two tumors, breast cancer and osteochondroma, with the occurrence of both lung cancer and osteochondroma in the same patients. Between 1973 and 1998, the same time-window that was used for assessing the odds ratio for an association between breast cancer and chondrosarcoma/enchondroma, there were 132636 cases of breast cancer and 16559 cases of lung cancer. In 28 women, both breast cancer and osteochondroma developed, whereas in lung cancer patients osteochondroma was reported 10 times. The odds ratio for the potential association of breast cancer and osteochondroma was 0.35 calculated as described previously $^{1}$ indicating that there is no statistically significant association for the concurrent development of breast cancer and osteochondroma. Furthermore, there is no record of an excess of breast cancer in MO families.

\section{BRCA1 and BRCA2 are not Responsible for this Syndrome}

To examine the possibility that these cartilaginous tumors are caused due to germline mutations in the BRCA1 and BRCA2 genes, we searched 41 BRCA1 and 12 BRCA2 families, with a proven inactivating mutation in one of these genes, from the files of the Foundation for Detection of Hereditary Tumors (http://www.nfdht. nl/STOET/) for cases with enchondroma and chondrosarcoma, both in the same patients as within the pedigree. However, no case of enchondroma or chondrosarcoma was found, implicating that the association between breast and cartilaginous tumors is not caused by germline mutations in BRCA1 and BRCA2 genes.

\section{Collection of Tumor Tissues}

Through PALGA, tumor tissue blocks from 61 patients with both breast cancer and a chondrosarcoma or enchondroma were requested. From five patients we did not receive any material. All received tumor specimens were reviewed histopathologically. Upon histological review, nine patients were excluded because a different diagnosis was determined for their presumed chondrosarcoma or enchondroma: myxoid chondrosarcoma $(n=1)$, synovial chondromatose $(n=1)$, subungual exostoses $(n=2)$, osteochondroma $(n=1)$, fibrochondroma $(n=1)$ and chondromas of the skin $(n=1)$, the vocal cord $(n=1)$ and the tendon sheath $(n=1)$.

From eight patients, we only received cartilaginous tumor specimens and from five patients, we only received breast cancer specimens. These 13 patients were also excluded since the diagnosis of the matching tumor could not be verified histologically. For 34 patients, both breast- and cartilaginous tumor specimens were included in this study. To our knowledge these patients are not related, but we 
cannot exclude this since patient samples were anonymized.

\section{Histological Examination}

The distributions of histological characteristics of our breast cancer specimens and the control group are listed in Table 3 . The data on the breast carcinomas in the control group were derived from the literature from a study to compare hereditary and sporadic breast cancer. ${ }^{16}$ Breast tumors within our series showed a significantly higher mitotic index $(P=0.001)$ when compared with the control group. Lymphocyte infiltrate and nuclear pleomorphism was less (respectively $P=0.025$ and 0.013) but after Bonferroni correction this was not significant anymore, because upon application of this correction the $P$-value should be smaller than 0.002. Other parameters, that is, tumor subtype, histological grade and presence of a component of carcinoma in situ were not significantly different.

Table 4 lists the comparison of histological features of the cartilaginous tumors in our series and of sporadic cases. All chondrosarcomas are of one common histological subtype being centrally localized whereas no chondrosarcomas secondary to osteochondroma with a peripheral localization were registered. This was compared with the distribution of enchondroma, primary and secondary chondrosarcoma reported ${ }^{17}$ showing a significant over-representation of central chondrosarcoma $(P=0.014)$. After Bonferroni correction this is not significant, but the complete absence of secondary

Table 3 Histopathological data in breast tumors associated with cartilaginous tumors vs controls

\begin{tabular}{|c|c|c|c|c|c|}
\hline \multirow[b]{2}{*}{ Cancer type } & \multicolumn{2}{|c|}{ Associated with cartilaginous tumors } & \multicolumn{2}{|c|}{ Control group 1} & \multirow[t]{2}{*}{ Significant different distribution } \\
\hline & & & & & \\
\hline Ductal & 35 & $90 \%$ & 1120 & $77 \%$ & \\
\hline Lobular & 3 & $8 \%$ & 142 & $10 \%$ & \\
\hline Tubular & 0 & $0 \%$ & 56 & $4 \%$ & \\
\hline Mucoid & 0 & $0 \%$ & 4 & $0,2 \%$ & \\
\hline Medullary & 0 & $0 \%$ & 20 & $1 \%$ & \\
\hline Ductal lobular & 0 & $0 \%$ & 38 & $3 \%$ & \\
\hline Other & 1 & $3 \%$ & 83 & $6 \%$ & \\
\hline \multicolumn{6}{|l|}{ Grade } \\
\hline 1 & 8 & $22 \%$ & 280 & $20 \%$ & \\
\hline 2 & 11 & $31 \%$ & 571 & $41 \%$ & \\
\hline 3 & 17 & $47 \%$ & 531 & $38 \%$ & \\
\hline \multirow{2}{*}{\multicolumn{6}{|c|}{ Tubules }} \\
\hline & & & & & \\
\hline$>90 \%$ & 5 & $14 \%$ & 93 & $7 \%$ & \\
\hline $50-90 \%$ & 4 & $11 \%$ & 315 & $23 \%$ & \\
\hline$<50 \%$ & 27 & $75 \%$ & 986 & $71 \%$ & \\
\hline \multicolumn{6}{|l|}{ Pleomorphism } \\
\hline 1 & 8 & $22 \%$ & 116 & $8 \%$ & \\
\hline 2 & 10 & $28 \%$ & 532 & $38 \%$ & \\
\hline \multirow[t]{2}{*}{3} & 18 & $50 \%$ & 747 & $54 \%$ & \\
\hline & & & & & $P=0.013^{*}$ \\
\hline \multicolumn{6}{|l|}{ Mitotic count } \\
\hline $0-4$ & 17 & $47 \%$ & 738 & $52 \%$ & \\
\hline $5-9$ & 2 & $6 \%$ & 219 & $16 \%$ & \\
\hline $10-19$ & 5 & $14 \%$ & 207 & $15 \%$ & \\
\hline $20-39$ & 11 & $31 \%$ & 140 & $10 \%$ & \\
\hline \multirow[t]{2}{*}{$40+$} & 1 & $3 \%$ & 103 & $7 \%$ & \\
\hline & & & & & $P=0.001^{* *}$ \\
\hline \multicolumn{6}{|l|}{ DCIS } \\
\hline Absent & 19 & $53 \%$ & 632 & $43 \%$ & \\
\hline Present & 17 & $47 \%$ & 831 & $57 \%$ & \\
\hline \multicolumn{6}{|l|}{ LCIS } \\
\hline Absent & 34 & $94 \%$ & 1382 & $94 \%$ & \\
\hline \multirow[t]{2}{*}{ Present } & 2 & $6 \%$ & 81 & $6 \%$ & \\
\hline \multirow{2}{*}{\multicolumn{6}{|c|}{ Lymphocyte infiltrate }} \\
\hline & & & & & \\
\hline Absent & 28 & $78 \%$ & 806 & $57 \%$ & \\
\hline Present & 9 & $25 \%$ & 610 & $43 \%$ & \\
\hline
\end{tabular}

${ }^{*}$ Not significant after Bonferroni correction.

${ }^{* *}$ Still significant after Bonferroni correction. 
Table 4 Histopathological data in cartilaginous tumors associated with breast tumors vs controls

\begin{tabular}{|c|c|c|c|c|c|}
\hline & \multicolumn{2}{|c|}{ Associated with breast tumors } & \multicolumn{2}{|c|}{ Control group 2} & \multirow[t]{2}{*}{ Significant different distribution } \\
\hline \multicolumn{5}{|l|}{ Tumor subtype } & \\
\hline Enchondroma & 18 & $53 \%$ & 616 & $29 \%$ & \\
\hline Peripheral chondrosarcoma & 0 & $0 \%$ & 216 & $10 \%$ & \\
\hline Central chondrosarcoma & 15 & $44 \%$ & 1079 & $50 \%$ & \\
\hline \multirow[t]{2}{*}{ Others } & 1 & $3 \%$ & 247 & $11 \%$ & \\
\hline & & & & & $P=0.014^{*}$ \\
\hline \multicolumn{6}{|l|}{ Grade } \\
\hline Enchondroma & 18 & $53 \%$ & 100 & $50 \%$ & \\
\hline Borderline chondrosarcoma & 5 & $15 \%$ & 19 & $10 \%$ & \\
\hline Chondrosarcoma grade I & 9 & $26 \%$ & 52 & $26 \%$ & \\
\hline Chondrosarcoma grade II & 1 & $3 \%$ & 22 & $11 \%$ & \\
\hline Chondrosarcoma grade III & 0 & $0 \%$ & 7 & $4 \%$ & \\
\hline \multicolumn{6}{|l|}{ Bones involved in enchondroma } \\
\hline Phalanx hand & 8 & $44 \%$ & 72 & $40 \%$ & \\
\hline Femur & 3 & $17 \%$ & 45 & $25 \%$ & \\
\hline Humerus & 2 & $11 \%$ & 23 & $13 \%$ & \\
\hline Rib & 2 & $11 \%$ & 14 & $8 \%$ & \\
\hline Phalanx foot & 2 & $11 \%$ & 9 & $5 \%$ & \\
\hline \multirow[t]{2}{*}{ Tibia } & 1 & $6 \%$ & 19 & $10 \%$ & \\
\hline \multirow{2}{*}{\multicolumn{6}{|c|}{ Bones involved in chondrosarcoma }} \\
\hline & & & & & \\
\hline Femur & 5 & $36 \%$ & 123 & $40 \%$ & \\
\hline Humerus & 4 & $29 \%$ & 44 & $14 \%$ & \\
\hline Phalanx hand & 1 & $7 \%$ & 16 & $5 \%$ & \\
\hline Metacarpel & 1 & $7 \%$ & 17 & $6 \%$ & \\
\hline Rib & 1 & $7 \%$ & 68 & $22 \%$ & \\
\hline Metatarsal & 1 & $7 \%$ & 6 & $2 \%$ & \\
\hline \multirow[t]{2}{*}{ Tibia } & 1 & $7 \%$ & 33 & $11 \%$ & \\
\hline & & & & & NS \\
\hline
\end{tabular}

${ }^{*}$ Not significant after Bonferroni correction.

peripheral chondrosarcomas is quite suggestive that this putative syndrome is specific for chondrosarcomas with a central localization. This confirms the lack of association between breast cancer and osteochondroma, the precursor of secondary chondrosarcoma. Other properties, that is, histological grade and location in the body, were not significantly different when compared with a control group of 200 women registered for chondrosarcoma or enchondroma in the files of the Netherlands Committee on Bone tumors (Table 4).

\section{Immunohistochemistry}

Table 5 summarizes the distributions of immunohistochemical features in breast cancer associated with cartilaginous tumors compared with nonselected controls from the literature. In order to identify any possible discerning feature, we compared staining intensity and number of cells stained separately for each antibody. However, because the controls were not obtained from the same laboratory we reduced the number of scoring categories to two, that is, negative and positive staining or high and low percentage of cells stained. There was a highly significant difference between estrogen receptor (ER) staining intensity in these breast cancers and controls $(P<0.001)$, which survives Bonferroni correction. In all, $69 \%$ of the breast cancers show moderate-to-strong ER intensity compared with $32 \%$ in controls. We also observed significant differences in the distribution of p53, both in terms of intensity and percentage of cells stained. In control tumor samples, $49 \%$ of cells show more than $1 \%$ positive cells staining for p53, compared with $91 \%$ of cells in associated breast cancer. Both p53 intensity and percentage of p53-positive cells are still significantly different after Bonferroni correction.

Her2 and E-cadherin protein expression did not differ with the controls. There were no suitable control groups available for P16 and P21 immunohistochemistry. Nuclear expression of the P16 protein was observed in $62 \%$ of tumors. Remarkably, we also observed cytoplasmic staining with the p16 antibody in $77 \%$ of the cases. In order to assess that this was not an artifact of the antibody a different antibody directed against p16 was used (16P04, also from Neomarkers), and this gave identical results. Only a minority of $6 \%$ showed negative staining for p21, which is concordant with previously reported results on nonselected breast tumors. ${ }^{24}$

The distribution of immunohistochemical staining in the cartilaginous tumors is summarized in Table 6. p53 staining was absent in the majority of the cases and mainly observed in chondrosarcomas and not in enchondromas as reported previously. ${ }^{25}$ p16 and p21 staining was positive in 87 and $67 \%$, 
Table 5 Immunohistochemical data for breast tumors

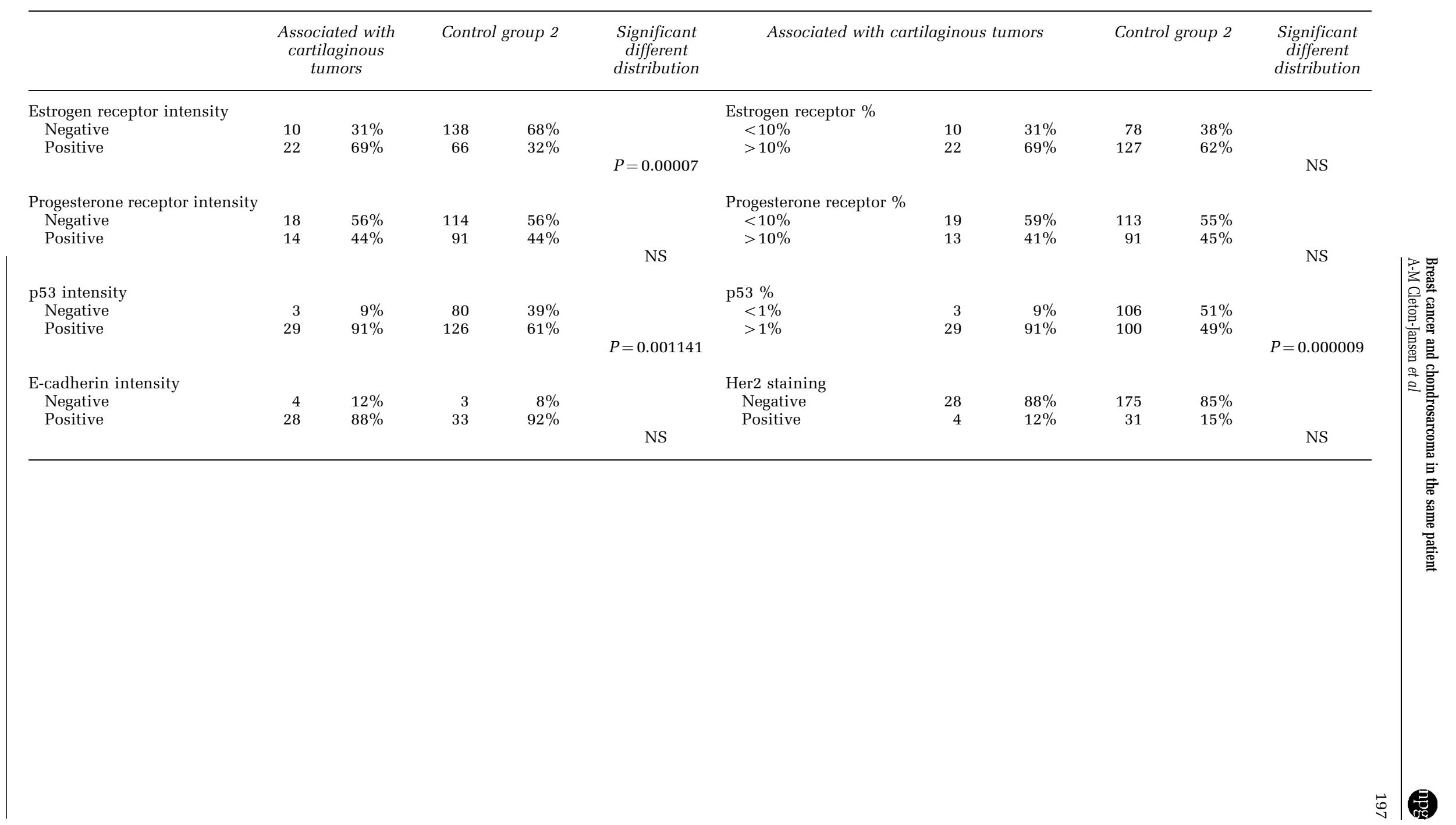


Table 6 Immunohistochemical data for cartilaginous tumors

\begin{tabular}{|c|c|c|c|c|c|}
\hline & \multicolumn{2}{|c|}{ Associated with breast tumors } & \multicolumn{2}{|c|}{ Control group 5} & \multirow[t]{2}{*}{ Significant different distribution } \\
\hline \multicolumn{5}{|l|}{ P16 intensity } & \\
\hline Negative & 3 & $13 \%$ & 11 & $10 \%$ & \\
\hline Weak & 1 & $4 \%$ & 37 & $33 \%$ & \\
\hline Moderate & 5 & $22 \%$ & 28 & $80 \%$ & \\
\hline \multirow[t]{2}{*}{ High } & 14 & $61 \%$ & 35 & $32 \%$ & \\
\hline & & & & & $P=0.015^{*}$ \\
\hline \multicolumn{6}{|l|}{ P16 \% } \\
\hline$<1$ & 3 & $13 \%$ & 11 & $10 \%$ & \\
\hline $1-25 \%$ & 6 & $26 \%$ & 41 & $37 \%$ & \\
\hline $26-50 \%$ & 11 & $48 \%$ & 32 & $29 \%$ & \\
\hline $51-75 \%$ & 3 & $13 \%$ & 14 & $13 \%$ & \\
\hline $76-100 \%$ & 0 & $0 \%$ & 13 & $12 \%$ & \\
\hline \multicolumn{6}{|l|}{ P21 staining } \\
\hline Negative & 10 & $33 \%$ & 3 & $5 \%$ & \\
\hline \multirow[t]{2}{*}{ Positive } & 20 & $77 \%$ & 52 & $95 \%$ & \\
\hline & \multirow{2}{*}{\multicolumn{5}{|c|}{ Control group 6}} \\
\hline P53 \%*** & & & & & \\
\hline$<25 \%$ & 12 & $40 \%$ & 73 & $80 \%$ & \\
\hline \multirow[t]{2}{*}{$>25 \%$} & 2 & $7 \%$ & 18 & $20 \%$ & \\
\hline & & & & & NS \\
\hline
\end{tabular}

${ }^{*}$ Not significant after Bonferroni correction.

$* *$ Still significant after Bonferroni correction.

${ }^{* * *}$ Chondrosarcomas only, no enchondromas.

respectively, in both chondrosarcomas and enchondromas. There was no significant difference in p16 expression when compared with controls (Van Beerendonk, manuscript in preparation). However, the absence of p21 staining is higher than in the control group, 33 vs $6 \%$ in nonselected cartilaginous tumors, a difference with a significant $P$-value of 0.001 .

\section{CHEK2 is not Involved in Tumors of this Putative Trait}

Because a rare genetic variant (1100delC) of the CHEK2 gene has recently been implicated in hereditary and sporadic breast cancer, ${ }^{26}$ we analyzed the breast tumors of our series for the occurrence of this variant. From the 34 breast cancers 24 cases yielded DNA of such quality that it was suitable for genetic analysis. However, none of these cases contained the 1100delC mutation, whereas the control sample showed a clear separate peak representing the variant.

CHEK2 protein expression was detected immunohistochemically and seven samples showed a somewhat decreased staining when compared with normal breast epithelial cells in the same section.

\section{Cluster Analysis}

Within the group of patients with an association between the occurrence of breast- and cartilaginous cancer there is expected to be a subset where cooccurrence is a coincidence. In an attempt to identify those 'sporadic cases', we performed an exploratory investigation using hierarchical cluster analysis on all clinical and immunohistochemical data collected on these patients. For this application, we used the data scored using the scoring system in Table 2, with more different scoring classes than used for comparing with controls, to identify possible subtle differences between tumors. A freeware computer program, originally developed to analyze complex cDNA microarray data, designated Cluster was used. ${ }^{23}$ The results of the hierarchical clustering are visualized using the associated program TreeView and shown in Figure 1. The breast tumors (Figure 1a) grouped in to two separate clusters. The largest group mainly consists of ER- and PR-positive and p53-negative breast cancer cases. Histological criteria are less informative. Chondrosarcomas and enchondromas (Figure 1b) show less pronounced clusters, probably because there are less data available. The cluster analysis for all data on both tumors in the same patient resembled the breast cancer clustering (Figure 1c).

\section{Discussion}

This study describes the phenotypic characterization of 34 patients with both a breast- and a cartilaginous tumor. One of the patients was male. Breast cancer in males is quite exceptional and occurs often in families with a germline mutation in BRCA2. ${ }^{27}$ However, we could not find any patient with a cartilaginous tumor in families with BRCA2 mutations, nor in BRCA1 pedigrees. If the presumed genetic trait that confers a risk for both breast and cartilaginous tumors is indeed caused by a 

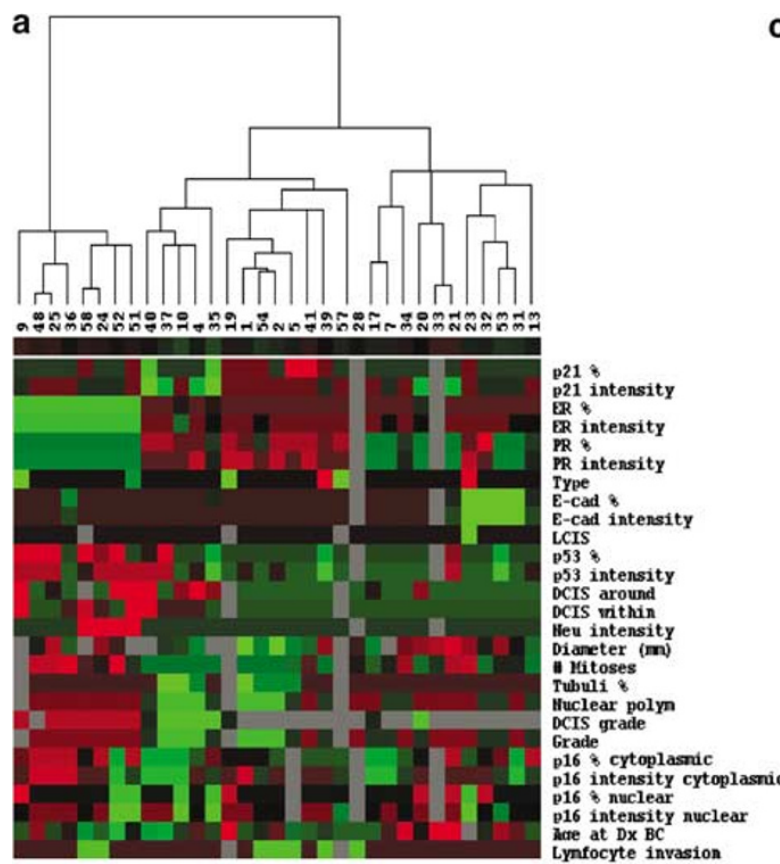

b

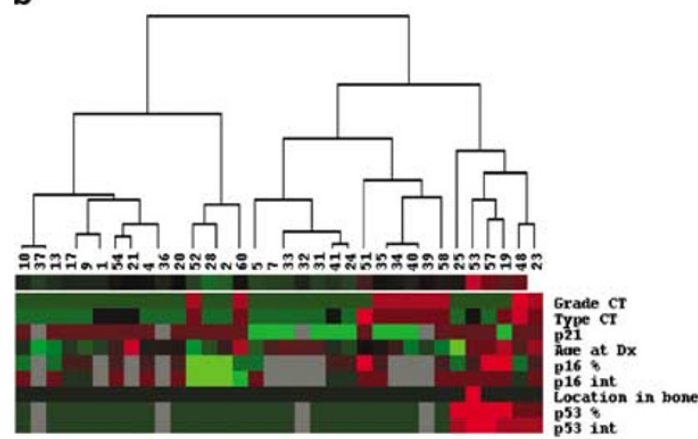

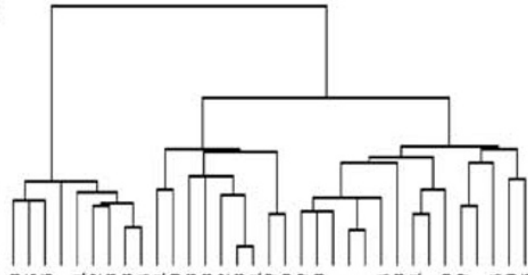
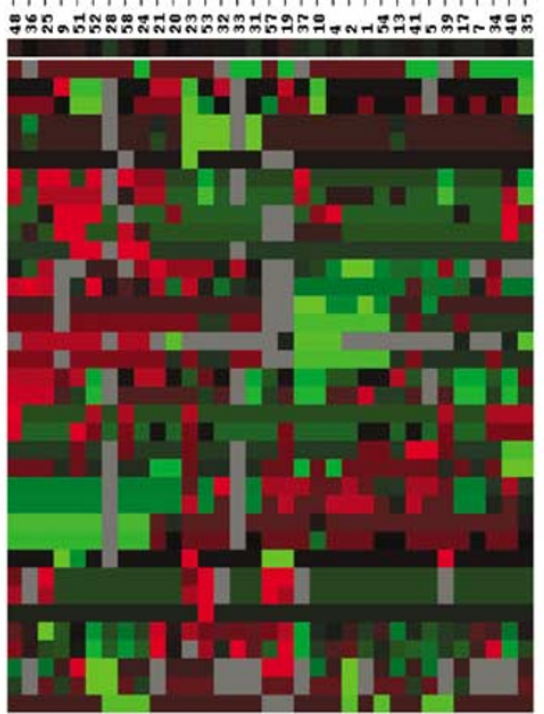
P21 CT
p16 8 nuclear BC
p16 int nuclear BC E-cad 8 BC LCIS p53 8 BC p53 int BC DCCS around OCIS within Heu int BC Dianeter Tubuli \& Huclear poly DCIS arade Grade BC p16 int cytoplassic BC Grade Cr Trpe CT

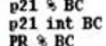
$\mathrm{PR}$ int $\mathrm{BC}$ ER \& BC $E R$ int $B C$ Tvee BC p53 p53 int CT Location in bon
hase at $\mathrm{Dx} \mathrm{CT}$ ane at $\mathrm{DX} C \mathrm{CT}$
haue at $\mathrm{Dx}$ BC p16 \& $\mathrm{cr}$ p16 int CT

0 1

Figure 1 (a) Cluster analysis on breast cancers (b) on chondro tumors and (c) on both tumors of the same patient. Genes are mean centered and normalized (0-1) with average linkage clustering and uncentered correlation is used as similarity metric. Bright green blocks represent the lowest value for each parameter, for example, negative immunostaining, low grade, low mitotic count, whereas bright red indicates the highest value. Colors in between are intermediate, with black being the mean value for each parameter. Gray blocks represent missing data.

particular germline mutation, BRCA1 or 2 are not likely as candidate genes.

To our knowledge chondrosarcoma is not part of the tumor spectrum of Li-Fraumeni or Li-Fraumenilike syndromes. In the Netherlands, only about 10 families are known to be affected by this trait and these cannot account for the 60 cases found by our association study. Moreover, in these families no chondrosarcomas are recorded to be present.

Another gene implicated in increased breast cancer risk is CHEK2, a cell-cycle checkpoint kinase that plays a role in DNA repair processes involving BRCA1 and p53. A specific variant (1100delC) that results in a truncated protein was found to occur at higher frequencies in patients with sporadic or familiar breast cancer. However, we did not find this variant in 25 patients with both breast and cartilaginous tumors tested, nor did we find loss of protein expression, which is often accompanying the 1100delC mutation. ${ }^{28}$ Other pathogenic variants of CHEK2 have not been identified in breast cancer. ${ }^{29}$ Apparently, loss of CHEK2 function does not play a role in this putative tumor syndrome.

In order to identify typical phenotypic characteristics of the tumors of these patients a histological and immunohistochemical survey was performed on 34 patients from which both cartilaginous- and breast tumor specimens were available. The breast cancer controls that were used in this study resulted from a multicenter pathological survey in which the same pathologist that examined the sections in our study was involved (MvdV), thereby ensuring uniformity of the scoring procedure. Also the immunohistochemical data for the cartilaginous tumors were examined uniformly and unlike the breast tumors generated in the same laboratory. For the breast 
tumors, the staining procedures may not be uniform and therefore, when comparing these data with controls a rough stratification was used, scoring tumors either as negative or positive for a particular antigen.

In comparison with the sporadic cases of breast cancer, this study shows a distinct histology and protein expression profile in cartilaginous tumorassociated breast cancer and therefore supports the existence of a new hitherto unrecognized hereditary trait.

The histological review of the breast cancer specimen displayed a higher mitotic count, reflecting an increased proliferation activity in cartilaginous tumor-associated breast cancer. Furthermore, we noted a trend towards less lymphocyte invasion and nuclear pleomorphism in these breast cancers. In our previous report, we already showed that the age of onset for breast cancer is 10 years earlier than in nonselected patients. ${ }^{1}$

Lakhani et $a l^{16}$ studied the morphological characteristics of familial breast tumors not attributable to BRCA1 and BRCA2. They presented these tumors as being of overall lower grade. ${ }^{16}$ However, the distribution of histological grade in the breast cancers in our series does not differ from the control group. Some reports have suggested that invasive lobular carcinoma and lobular carcinoma in situ is associated with a higher familial risk than other invasive or in situ subtypes. ${ }^{30,31}$ However, the lobular subtype is under-represented in BRCA1and BRCA2-associated breast cancer. Also in our study, we do not find an increased frequency of invasive lobular breast carcinomas or lobular carcinoma in situ.

Immunohistochemical analysis of these breast cancer specimens showed significantly higher intensity of the estrogen receptor (ER) staining, however, no higher number of ER-positive cells. Because these tumors show high expression of ER, they may be more susceptible for estrogen-antagonist therapy. Interestingly, estrogen metabolism also plays a role in cartilage, since it initiates the pubertal growth spurt, causes closing of the growth plate and augments bone accrual during puberty. ${ }^{32}$ Unfortunately, the antibody used for ER in breast cancer failed to stain sections of cartilaginous tissue, probably due to the excess of extracellular matrix.

Aberrant staining of p16 in the cytoplasm has been described previously and was shown to be associated with a more malignant phenotype,$^{33}$ but only in a small subset of $9 \%$ of the cases. The p16positive breast cancer cases described here showed markedly more cytoplasmic staining in $77 \%$ $(n=24)$. Because cytoplasmic p16 staining was not recorded for the control series used, we could not calculate whether this difference was significant.

We also observed a distinct histological feature within breast cancer-associated cartilaginous tumors, which all originated centrally in the bone. This finding is remarkable because within the general population $15 \%$ of all chondrosarcomas are peripherally localized, suggesting a specific genetic mechanism in the development of these breast cancer-associated cartilaginous tumors. This hypothesis is corroborated by Bovee et al, ${ }^{11}$ who described that different genetic mechanisms are operational in central and peripheral chondrosarcomas. No correlation could be made with histological grade, because of the relatively small numbers of mostly low-grade chondrosarcomas. The benign cartilaginous lesions $(n=18)$ are all enchondromas, which are considered as precursor for central, but not for peripheral chondrosarcomas. We have shown here that osteochondromas, the precursor of peripheral chondrosarcoma are not associated with a higher incidence of breast cancer in the same patient, thereby providing additional evidence that this presumed genetic trait is characterized by central chondrosarcoma and its potential precursor only.

p21 staining was negative in more cases than in the control series of cartilaginous tumors. This may be a distinguishing property of breast cancerassociated cartilaginous tumors. Remarkably, all but one patient with p21-negative cartilaginous tumors have ER-positive breast cancers, which are from the patient group suspected to belong to the putative genetic trait. p21 expression is regulated by p53 and none of the 6 p53-positive cartilaginous tumors show p21 expression, suggesting that these cases have a nonfunctional p53 protein. Indeed, p53 inactivating mutations have been reported to occur in chondrosarcoma. ${ }^{34}$

The normal lifetime risk for a woman to develop breast cancer is one in 12 in Europe and therefore it is likely that our series is also containing patients who have both tumors by chance. In order to further elucidate the genetics of this trait it is important to identify those patients. The cluster analysis performed on all data indeed identifies two different clusters; however, the patients are not clustering according to all significantly different features. Therefore, it cannot be concluded that this cluster analysis provides a means to distinguish sporadic cases from patients that belong to this genetic trait. The smallest cluster on the left in Figure 1a and c contains ER- and PR-negative tumors often with p53 overexpression, a DCIS component and of high histological grade. The largest cluster on the right in Figure 1a and c contains ER-positive, and mostly p53-negative breast tumors. According to the casecontrol comparisons of our study a positive ER is prevalent in breast cancers of patients with cartilaginous cancer, thereby suggesting that the largest cluster contains patients who may belong to the putative genetic trait. The only male breast cancer patient in the series clustered within this cluster. Also p53 overexpression was significantly higher when compared with controls but p53-positive breast cancers are primarily in the other cluster. However, the distribution of p53-positive cancers is 
less clearcut. The other markers in the associated breast tumors that were different when compared to controls that is, the higher mitotic index, the lower incidence of lymphocyte and nuclear pleomorphism infiltrate did not cluster with the ER-positive patients. The latter two parameters were not significant after Bonferroni correction.

In summary, we have investigated the phenotypic characteristics of breast and cartilaginous tumors occurring in the same patients and identified features that are indeed indicative of a specific trait. A positive ER and early age of onset characterizes breast tumors whereas central origin and loss of p21 protein is typical for the cartilaginous tumors that are associated. At the moment, no pedigrees are available where both tumor types cluster in different members. This suggests that the genetic defect underlying this trait is not a single gene, but involves several separate alleles that when shared in an individual result in this phenotype.

\section{Acknowledgement}

We want to acknowledge M Casparie from PALGA, who was very helpful in gathering our tumor specimens, LCJM van den Broek who performed the immunohistochemistry, $M$ van Puijenbroek and $\mathrm{H}$ van Beerendonk who designed and performed CHEK2 variant analysis, Drs P Devilee and R Brohet who provide the data on BRCA1 and 2 families and Dr JVMG Bovée who examined the control series. This study was supported by a grant from the Optimix Foundation for Fundamental Research.

\section{References}

1 Odink AE, van Asperen CJ, Vandenbroucke JP, et al. An association between cartilaginous tumours and breast cancer in the national pathology registration in The Netherlands points towards a possible genetic trait. J Pathol 2001;193:190-192.

2 Breast Cancer Linkage Consortium. Pathology of familial breast cancer: differences between breast cancers in carriers of BRCA1 or BRCA2 mutations and sporadic cases. Lancet 1997;349:1505-1510.

3 Lakhani SR, Van de Vijver MJ, Jacquemier J, et al. The pathology of familial breast cancer: predictive value of immunohistochemical markers estrogen receptor, progesterone receptor, HER-2, and p53 in patients with mutations in BRCA1 and BRCA2. J Clin Oncol 2002;20:2310-2318.

4 Van 't Veer LJ, Dai H, Van de Vijver MJ, et al. Gene expression profiling predicts clinical outcome of breast cancer. Nature 2002;415:530-536.

5 Hedenfalk I, Duggan D, Chen Y, et al. Gene-expression profiles in hereditary breast cancer. N Engl J Med 2001;344:539-548.

6 Springfield DS, Gebhardt MC, McGuire MH. Chondrosarcoma: a review. J Bone Joint Surg [Am] 1996; 78A:141-149.
7 Mulder JD, Schütte HE, Kroon HM, et al. Radiologic Atlas of Bone Tumors. Elsevier: Amsterdam, 1993.

8 WHO 2002. Cartilage tumours. In: Fletcher CDM, Unni KK, Mertens F (eds). World Health Organization Classification of Tumours. Pathology and Genetics. Tumours of Soft Tissue and Bone. IARC Press: Lyon, 2002, pp 234-257.

9 Bertoni F, Bacchini P, Hogendoorn PCW. Chondrosarcoma. In: Fletcher CDM, Unni KK, and Mertens F (eds). World Health Organisation Classification of Tumours. Pathology and Genetics of Tumours of Soft Tissue and Bone. IARC Press: Lyon, 2002, pp 247-251.

10 Bovee JVMG, Sciot R, Dal Cin P, et al. Chromosome 9 alterations and trisomy 22 in central chondrosarcoma: a cytogenetic and DNA flow cytometric analysis of chondrosarcoma subtypes. Diagn Mol Pathol 2001; 10:228-236.

11 Bovee JVMG, Cleton-Jansen AM, Kuipers-Dijkshoorn $\mathrm{N}$, et al. Loss of heterozygosity and DNA ploidy point to a diverging genetic mechanism in the origin of peripheral and central chondrosarcoma. Genes Chromosomes Cancer 1999;26:237-246.

12 Bovee JVMG, Royen Mv, Bardoel AFJ, et al. Nearhaploidy and subsequent polyploidization characterize the progression of peripheral chondrosarcoma. Am J Pathol 2000;157:1587-1595.

13 Schmale GA, Conrad EU, Raskind WH. The natural history of hereditary multiple exostoses. J Bone Joint Surg [Am] 1994;76A:986-992.

14 Wicklund LC, Pauli RM, Johnston D, et al. Natural history study of hereditary multiple exostoses. Am J Med Genet 1995;55:43-46.

15 Wuyts W, Van Hul W, De Boulle K, et al. Mutations in the EXT1 and EXT2 genes in hereditary multiple exostoses. Am J Hum Genet 1998;62:346-354.

16 Lakhani SR, Gusterson BA, Jacquemier J, et al. The pathology of familial breast cancer: histological features of cancers in families not attributable to mutations in BRCA1 or BRCA2. Clin Cancer Res 2000; 6:782-789.

17 Mirra JM. Bone Tumors. Clinical, Radiologic, and Pathologic Correlations. Lea \& Febiger: Philadelphia, 1989.

18 Oshiro Y, Chaturvedi V, Hayden D, et al. Altered p53 is associated with aggressive behavior in chondrosarcoma; a long term follow-up study. Cancer 1998;83: 2324-2334.

19 WHO 1981. Histologic Typing of Breast Tumours. WHO: Geneva, 1981.

20 Evans HL, Ayala AG, Romsdahl MM. Prognostic factors in chondrosarcoma of bone. A clinicopathologic analysis with emphasis on histologic grading. Cancer 1977;40:818-831.

21 Bovee JVMG, Van den Broek LJCM, De Boer WI, et al. Expression of growth factors and their receptors in adamantinoma of long bones and the implications for its histogenesis. J Pathol 1998;184:24-30.

22 Bovee JVMG, Van den Broek LJCM, Cleton-Jansen AM, et al. Up-regulation of PTHrP and Bcl-2 expression characterizes the progression of osteochondroma towards peripheral chondrosarcoma and is a late event in central chondrosarcoma. Lab Invest 2000;80: 1925-1933.

23 Eisen MB, Spellman PT, Brown PO, et al. Cluster analysis and display of genome-wide expression patterns. Proc Natl Acad Sci USA 1998;95: 14863-14868. 
24 Thor AD, Liu S, Moore DH, et al. p(21WAF1/CIP1) expression in breast cancers: associations with p53 and outcome. Breast Cancer Res Treat 2000;61:33-43.

25 Coughlan B, Feliz A, Ishida T, et al. p53 expression and DNA ploidy of cartilage lesions. Hum Pathol 1995;26:620-624.

26 Meijers-Heijboer $\mathrm{H}$, van den OA, Klijn J, et al. Lowpenetrance susceptibility to breast cancer due to CHEK2 $\left({ }^{*}\right) 1100 d e l C$ in noncarriers of BRCA1 or BRCA2 mutations. Nat Genet 2002;31:55-59.

27 Wooster R, Bignell G, Lancaster J, et al. Identification of the breast cancer susceptibility gene BRCA2. Nature 1995;378:789-792.

28 Oldenburg RA, Kroeze-Jansma K, Kraan J, et al. The CHEK2*1100delC variant acts as a breast cancer risk modifier in non-BRCA1/BRCA2 multiple-case families. Cancer Res 2003, (in press).

29 Schutte M, Seal S, Barfoot R, et al. Variants in CHEK2 other than 1100delC do not make a major contribution to breast cancer susceptibility. Am J Hum Genet 2003;72:1023-1028.

30 Cannon-Albright LA, Thomas A, Goldgar DE, et al. Familiality of cancer in Utah. Cancer Res 1994;54: 2378-2385.

31 Rosen PP, Lesser ML, Senie RT, et al. Epidemiology of breast carcinoma III: relationship of family history to tumor type. Cancer 1982;50:171-179.

32 van der Eerden BC, Karperien M, Wit JM. The estrogen receptor in the growth plate: implications for pubertal growth. J Pediatr Endocrinol Metab 2001;14(Suppl 6):1527-1533.

33 Emig R, Magener A, Ehemann V, et al. Aberrant cytoplasmic expression of the p16 protein in breast cancer is associated with accelerated tumour proliferation. Br J Cancer 1998;78:1661-1668.

34 Toguchida J, Yamaguchi T, Ritchie B, et al. Mutation spectrum of the p53 gene in bone and soft tissue sarcomas. Cancer Res 1992;52:6194-6199.

\section{Electronic-Database Information}

SISA (simple interactive statistical analysis) website: http://home.clara.net/sisa/bonhlp.htm Cluster and TreeView programs are available at http://rana.lbl.gov/EisenSoftware.htm 